
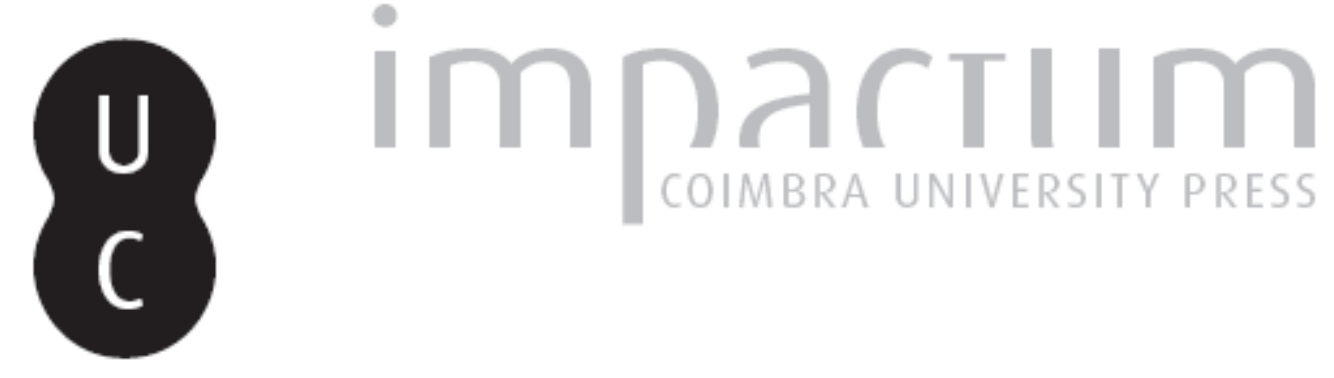

O uso de geotecnologias para a análise da gestão de risco prospectiva: as doenças e a ocorrência das inundações por precipitações pluviométricas no estado de Santa Catarina. Sul do Brasil

Autor(es): $\quad$ Diesel, Lilian Elizabeth; Ometto, Jean Pierre Henry Balbaud

Publicado por: Associação Portuguesa de Riscos, Prevenção e Segurança; Imprensa

URL

persistente: URI:http://hdl.handle.net/10316.2/39726

DOI: ～DOI:https://doi.org/10.14195/1647-7723_23_2

Accessed : $\quad$ 26-Apr-2023 09:32:33

A navegação consulta e descarregamento dos títulos inseridos nas Bibliotecas Digitais UC Digitalis, UC Pombalina e UC Impactum, pressupõem a aceitação plena e sem reservas dos Termos e Condições de Uso destas Bibliotecas Digitais, disponíveis em https://digitalis.uc.pt/pt-pt/termos.

Conforme exposto nos referidos Termos e Condições de Uso, o descarregamento de títulos de acesso restrito requer uma licença válida de autorização devendo o utilizador aceder ao(s) documento(s) a partir de um endereço de IP da instituição detentora da supramencionada licença.

Ao utilizador é apenas permitido o descarregamento para uso pessoal, pelo que o emprego do(s) título(s) descarregado(s) para outro fim, designadamente comercial, carece de autorização do respetivo autor ou editor da obra.

Na medida em que todas as obras da UC Digitalis se encontram protegidas pelo Código do Direito de Autor e Direitos Conexos e demais legislação aplicável, toda a cópia, parcial ou total, deste documento, nos casos em que é legalmente admitida, deverá conter ou fazer-se acompanhar por este aviso.

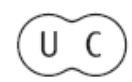






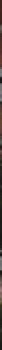

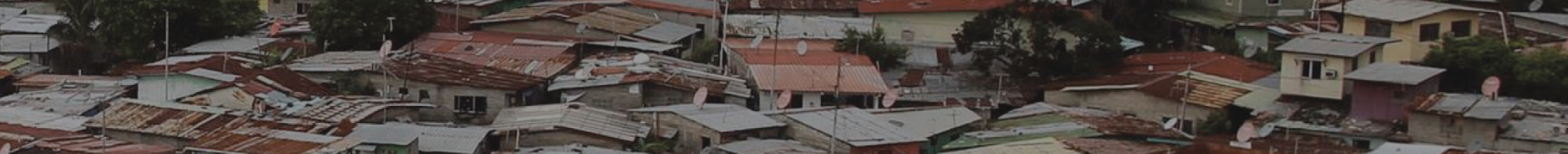

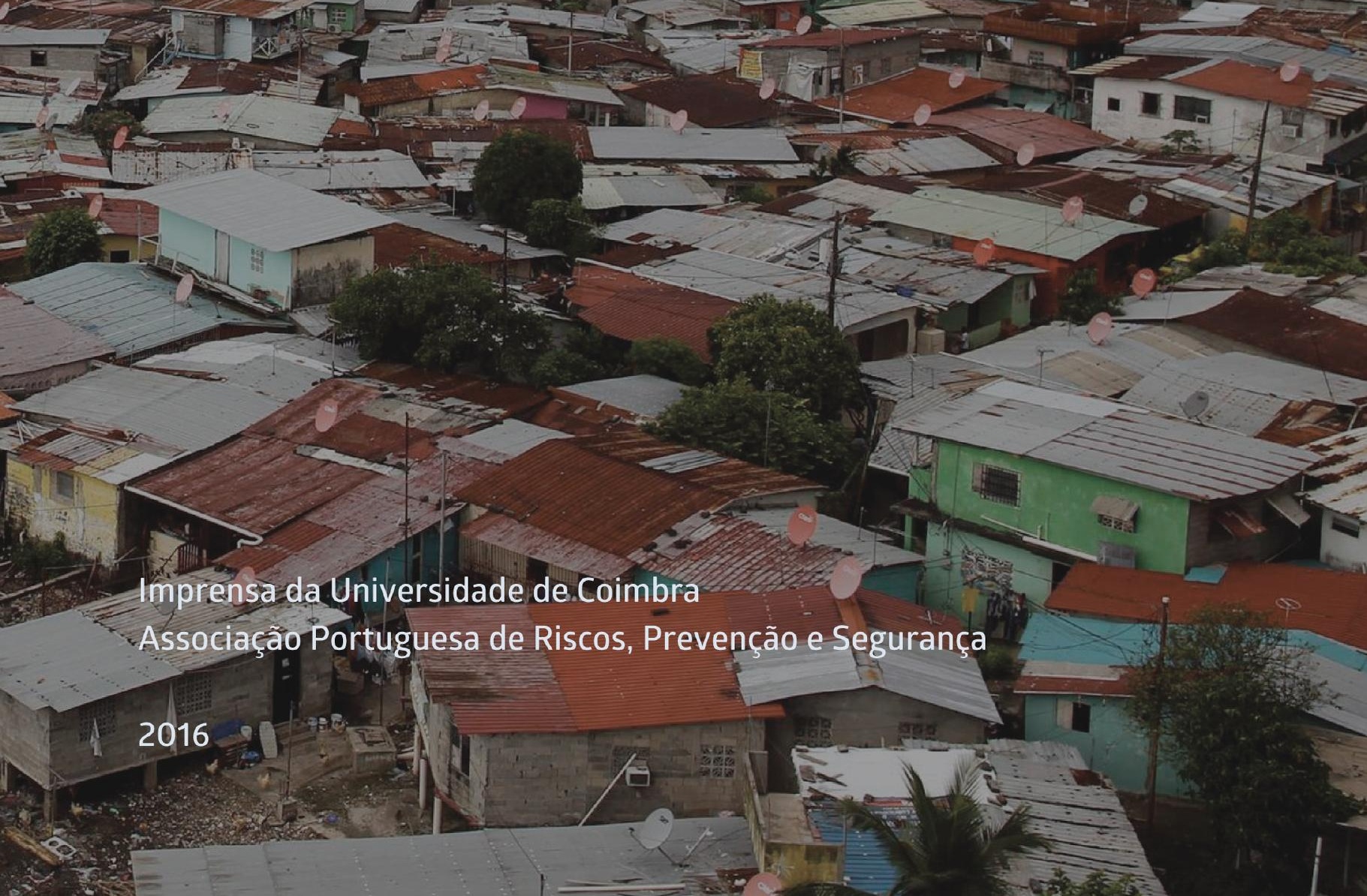




\section{O USO DE GEOTECNOLOGIAS PARA A ANÁLISE DA GESTÃO DE RISCO PROSPECTIVA: AS DOENÇAS E A OCORRÊNCIA DAS INUNDAÇ̃̃ES POR PRECIPITAÇÕES PLUVIOMÉTRICAS NO ESTADO DE SANTA CATARINA - SUL DO BRASIL*}

\section{THE USE OF GEOTHECHNOLOGY FOR THE ANALYSIS OF PROSPECTIVE RISK MANAGEMENT: DISEASES AND THE OCCURRENCE OF FLOODING BY RAINFALL IN SANTA CATARINA STATE - SOUTH OF BRAZILL}

Lilian Elizabeth Diesel Instituto de Pesquisas Espaciais - INPE lidiesel@gmail.com

Jean Pierre Henry Balbaud Ometto Instituto de Pesquisas Espaciais - INPE jean.ometto@inpe.br

RESUMO

A presente pesquisa objetiva a análise exploratória dos dados de doenças infecciosas, como síndrome diarreica aguda e a leptospirose, com as inundações por precipitações pluviométricas ocorridas no estado de Santa Catarina, durante o período de 2008 a 2012. Demonstra esta pesquisa que o uso da geotecnologia associada à gestão de risco prospectivo contribui para a definição de intervenções com o propósito de minimizar as ocorrências das doenças em casos de inundações por precipitações pluviométricas.

Palavras-chave: Gestão de risco, geotecnologias, epidemiologia dos desastres, inundações.

\section{ABSTRACT}

This research aims the exploratory data analysis of infectious diseases, such as acute diarrhea syndrome and leptospirosis with flooding by rainfall that occurred in the State of Santa Catarina, during the period from 2008 to 2012. The research shows that the use of geotechnology associated with prospective risk management contributes to the definition of interventions in order to minimize the occurrence of diseases in cases of floods by rainfall.

Keywords: Risk management, geotechnology, epidemiology of disasters, flooding.

\section{RESUMEN}

El uso de geotecnologías para el análisis de la gestión de riesgo prospectivo: las enfermedades y la ocurrencia de inundaciones por precipitaciones pluviométricas en el Estado de Santa Catarina - Sur de Brasil - La presente investigación objetiva el análisis exploratorio de los datos de enfermedades infecciosas, como síndrome de diarrea aguda y leptospirosis, con las inundaciones por precipitaciones pluviométricas ocurridas en el estado de Santa Catarina, durante el período de 2008 a 2012. Esta investigación demuestra que el uso de la geotecnología asociada a la gestión de riesgo prospectivo contribuye para la definición de intervenciones con el propósito de minimizar las ocurrencias de las enfermedades en casos de inundaciones por precipitaciones pluviométricas.

Palabras clave: Gestión del riesgo, geotecnología, epidemiología de los desastres, inundaciones.

\section{RESUMÉ}

L'utilisation de la géotechnologie pour l'analyse de la gestion prospective des risques: l'apparition des inondations pluviales et les maladies dans l'état de Santa Catarina - Sud du Brésil - Cette recherche vise l'analyse des données des maladies infectieuses telles que le syndrome de la diarrhée aiguë, et la leptospirose, recensées lors des épisodes d'inondations pluviales qui se sont produites dans l'état de Santa Catarina, au cours de la période de 2008 à 2012 . La recherche démontre que l'utilisation de la géotechnologie associée à la gestion prospective de risques contribue à la définition des interventions. Elle permet aussi de minimiser l'apparition des maladies en cas d'inondations par la pluie.

Mots-clé: La gestion des risques, géotechnologie, épidémiologie des catastrophes, inondations.

* O texto deste artigo corresponde a uma comunicação apresentada no I Seminário da Rede Incêndios-Solo e I Simpósio Ibero-Afro-Americano de Riscos, tendo sido submetido em 24-11-2015, sujeito a revisão por pares a 18-03-2016 e aceite para publicação em 31-03-2016.

Este artigo é parte integrante da Revista Territorium, n. ${ }^{\circ} 23,2016,{ }^{\circ}$ RIscos, ISSN: 0872-8941. 


\section{Introdução}

As ocorrências de desastres naturais, no caso as inundações, encontram-se relacionadas com o aumento de doenças infecciosas como a síndrome da diarreia aguda (SDA), a leptospirose, as infecções respiratórias agudas, os sarampos, o tétano, dentre outras. Para que seja possível realizar uma análise das ocorrências das inundações relacioandas ao aumento da incidência dos casos de SDA e Leptospirose, objetos de estudo da presente pesquisa, faz-se necessário o uso das geotecnologias, com o propósito de aumentar a sua eficácia, uma vez que tanto as ocorrências de inundações quanto as doenças infecciosas crescem de forma considerável.

As geotecnologias permitem a realização de uma grande variedade de análises, inclusive as análises espaciais, assim como, do armazenamento de grandes bancos de dados, neste caso, de desastres e saúde, permitindo, inclusive, o desenvolvimento da gestão de risco prospectiva em todas as suas fases, como: análise de prevenção, monitoramento e resposta. (L. Diesel, 2013; D. Cerutti e M. Oliveira, 2011; L. Agredo, 2011).

A gestão de risco prospectiva para a ocorrência das inundações por precipitações pluviométricas e sua relação com os casos de SDA e Leptospirose, devem, no mínimo, permitir a identificação do fenômeno através de sua caracterização, localização e causas, além das alternativas para intervenção, no caso, as tomadas de decisões. As informações geradas com foco em tomadas de decisões devem permitir aos gestores uma rápida resposta objetivando a minimização dos casos das doenças infecciosas como a SDA e a leptospirose.

Em se tratando da gestão de risco prospectiva para desastres e saúde, é uma necessidade urgente a utilização de todas as possibilidades para melhorar a sua eficácia tendo como base as crescentes ameaças às populações.

\section{Objetivo}

Esta pesquisa objetivou a análise da gestão de risco prospectiva através de análises exploratórias dos dados de inundações por precipitações pluviométricas e dos dados de ocorrência de doenças infecciosas como a síndrome diarreica aguda e a leptospirose no estado de Santa Catarina - Sul do Brasil, bem como, da aplicação das geotecnologias para a distribruição espacial - mapeamento.

\section{Revisão bibliográfica}

O uso das geotecnologias para a gestão de risco prospectiva contribuem para a definição de medidas de intervenção no que se referem às questões de saúde pública e desastres, no caso desta pesquisa, com proposição de auxiliar na minimização da ocorrência das doenças. Muitos estudos já foram desenvolvidos por outros autores e aplicadas para diversas áreas do conhecimento como: transportes, gestão territorial, segurança pública dentre outras. (E. Souza, et al., 2014; L. Diesel, 2013; M. Freitas e L. Cunha, 2013; M. Fuentes Vallejo et.al., 2015).

As geotecnologias aplicadas no caso das doenças infecciosas, a exemplo da SDA e da leptospirose permite que se faça o entendimento da gestão de risco prospectiva. Sobre a gestão da risco prospectiva é possível destacar sua definição como sendo uma prática que procura evitar erros passados (D. Cerutti e M. Oliveira, 2011). Da mesma forma, este tipo de gestão estabelece uma relação direta e indireta com as fases do planejamento para minimizar o risco e sua evolução. (D. Cerutti e M. Oliveira, 2011)

Outra definição para a gestão de risco prospectiva (L. Agredo, 2011), é que a mesma tem condições de identificar e permitir a realização de previsão e antecipação de risco futuro. Este tipo de gestão antecipa o risco com base na investigação de eventos já ocorridos.

As ocorrências das inundações por precipitações pluviométricas no estado de Santa Catarina, já são fatos bastante conhecidos, com seguidas e intensas ocorrências, causando diversos tipos de danos às comunidades afetadas. Os tipos de inundações mais comuns ocorridas na àrea de estudo são as graduais e as bruscas. As inundações graduais são entendidas como sendo a elevação do nível dos rios de forma paulatina e previsível, mantendo-se em cheia durante certo período de tempo, e, a seguir escoam-se de forma gradual (A. Castro, 2003). A inundação brusca é de ocorrência súbita, com elevações rápidas dos níveis dos rios, gerando um transbordamento das águas, que escoam de forma rápida (A. Castro, 2003).

As ocorrências das inundações, sejam elas bruscas ou graduais, sempre causam consequências para as populações. As consequências mais comuns são as ocorrências de doenças infecciosas. Das doenças relacionadas às inundações destacamos à Sindrome Diarreica Aguda (SDA) e a Leptospirose.

A SDA relaciona-se com a inundação, pois, existe uma maior vulnerabilidade por exposição a água contaminada através do processo da inundação. A diarreia é considerada como a principal causa de mortes em desastres ou abrigos, e, sua propagação, ocorre pela contaminação principalmente de material fecal e pela falta de higiene. (R. Fagundes, 2015)

A leptospirose, outra doença infecciosa, que apresenta relação com as inundações, possui mais de 20 espécies de espiroquetas, nos mais variados hospedeiros. Os hospedeiros mais comuns são os ratos, outros roedores, 
bem como, o gado e os animais domésticos. A transmissão ocorre pelo contato direto com os animais, com o solo úmido, a lama e a água, estes se contaminados pela urina de roedores portadores da bactéria. A contaminação se dá principalmente pela ingestão de alimentos ou água contaminda, através do contato com a mucosa ou a pele. $\mathrm{O}$ homem é considerado como hospedeiro acidente, e raramente transmite a doença.(D. Winckler, 2015; R. Guimarães, et.al., 2014)

Em concordancia com os autores estudados, foi possível identificar que para uma gestão de risco prospectiva eficiente é necessária a aplicação de geotecnologias, pois estas permitem o cruzamento de diversas informações oriundas de distintas instituições, contribuindo para um melhor planejamento de ações que minimizem os erros passados.

\section{Materiais e métodos}

Para o estudo da gestão de risco prospectiva, faz-se necessaria a realização da revisão bibliográfica, a experimentação e a descrição dos dados históricos. Estes são os componentes para o desenvolvimento de uma abordagem científica conforme ponderam alguns autores. (S. Vergara, 2007; E. Lakatos e M. Marcon, 2007).

As abordagens descritas permitem aprofundar 0 conhecimento sobre o tema abordado e definir o tipo de pesquisa a ser realizada. Portanto, optou-se pela pesquisa experimental, com a aplicação das geotecnologias para a análise da gestão de risco prospectiva, com base nas análises das ocorrências de doenças e inundações, no estado de Santa Catarina - Sul do Brasil.

As etapas de desenvolvimento desta pesquisa foram: construção da fundamentação teórica, desenho da pesquisa, coleta e análise dos dados, e a aplicação da geotecnologia através do mapeamento-distribuição espacial.

\section{Fundamentação teórica}

Esta etapa foi construída com base em revisão bibliográfica com os seguintes temas: geotecnologias, gestão de risco prospectiva, desastres - inundações, doenças e vulnerabilidades. Com base no que foi identificado na revisão bibliográfica definiu-se o desenho da pesquisa.

\section{Desenho da pesquisa}

Durante o processo de revisão bibliográfica foram identificadas as necessidades de se trabalhar com os dados de doenças infecciosas como a Síndrome Diarreica Aguda e a Leptospirose. Estas doenças se apresentam de forma distinta em seu desenvolvimento durante os eventos de precipitações pluviométricas intensas, ou seja, nas ocorrências de inundações.
Desta forma, optou-se para esta pesquisa, pela análise dos dados de ocorrência tanto das doenças quanto das inundações e o mapeamento - distribuição espacial-, com base em eventos já ocorridos.

\section{Coleta e análise dos dados}

A coleta de dados deu-se através do inventário dos mesmos junto da Secretaria de Defesa Civil do Estado de Santa Catarina, e a obtenção dos dados de doenças junto da Vigilância Epidemiológica do Estado de Santa Catarina.

O inventário de dados de inundações ocorridas no estado de Santa Catarina ocorreu através de pesquisa de campo, com visitas à Secretaria de Defesa Civil do Estado de Santa Catarina, no período de julho de 2014 até fevereiro de 2015. Os dados foram obtidos na forma manual, assim como através de escaneamento de AVADANS (boletim de avaliação de danos) que depois foram convertidos em documentos Excel para a construção do banco de dados de desastres. Este banco de dados é composto das seguintes variáveis: municípios, data de ocorrência, tipo de desastre, número de afetados, número de desabrigados, número de desalojados, número de feridos, número de enfermos, número de mortes, área afetada, danos humanos, danos materiais, danos ambientais, danos agropecuários, danos econômicos, danos sociais, intensidade do desastre e o tipo de decreto.

A coleta dos dados referentes às doenças - síndrome diarreica aguda e leptospirose-, foram obtidos diretamente da Vigilância Epidemiológica do Estado de Santa Catarina. Os dados que compõe este conjunto de informações sãp relativos ao: mês de ocorrência, município de notificação, município de residência e o total de doenças.

A base cartográfica utilizada para o mapeamento das análises pertence ao IBGE, atualizada em 28/07/2015, em formato digital shapefile, contendo canevá cartográfico em coordenadas UTM (Universal Transversal de Mercator), no Sistema SIRGAS 2000 (South American Geocentric Reference System) com representações equivalentes às escalas de 1:100.000 e 1:50.000. Além do canevá, contém os limites do estado de Santa Catarina e os limites municipais.

As análises realizadas nesta pesquisa são do tipo exploratória, de apoio à decisão e distribuição espacial - mapeamento. As análises exploratórias dos dados foram as médias pluviométricas, os dados absolutos, as taxas de incidências (por 100.000 habitantes) e o risco relativo. Essas análises foram realizadas por ano e por mês, no período de estudo de 2008 até 2012.

O risco relativo foi realizado mês a mês, estimados para a ocorrência de síndrome diarréica aguda e leptospirose e a média de precipitação pluviométrica no mês anterior 
aos casos. A referência utilizada para estimar o risco relativo foi o mês de junho, que possui a menor média de precipitação pluviométrica do ano.

As análises de apoio à decisão, e que fazem parte da análise da gestão de risco prospectiva, tem como base o resultado das análises demonstradas nesta pesquisa, assim como, da distribuição espacial - mapeamento.

\section{Resultados}

Os resultados obtidos nas análises podem ser observados nas figuras que seguem. Na fig. 1 e na fig. 2, encontramse as médias de precipitações pluviométricas por ano e por mês, segundo o período de estudo. Na fig.2 e na fig. 3 encontram-se as incidências anuais e mensais, tanto para a síndrome diarreica aguda quanto para a leptospirose.

Observa-se na fig. 1 que as maiores médias anuais de precipitações pluviométricas para o estado de Santa Catarina, deram-se nos anos de 2011 e 2010, seguidos do ano de 2008. As médias das precipitações pluviométricas mensais representativas para os anos de 2008 até 2012, foram identificadas no mês de janeiro, este como sendo o mais chuvoso, apresentando uma média de $232,73 \mathrm{~mm}$, seguido do mês de outubro com $198,47 \mathrm{~mm}$ e fevereiro com 181,66mm, conforme demonstrado na fig. 2 .

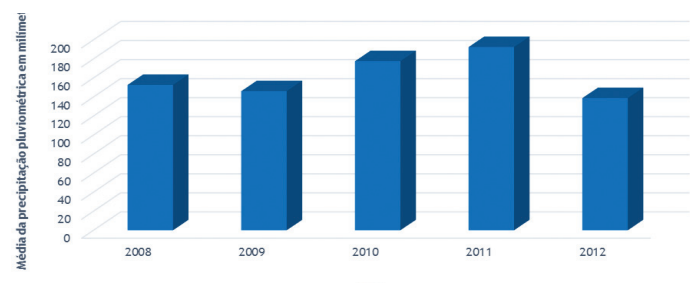

Fig. 1 - Médias anuais das precipitações pluviométricas no período de 2008 até 2012. Estado de Santa Catarina - Sul do Brasil.

Fig. 1 - Annual averages of rainfall from 2008 to 2012. State of Santa Catarina - South of Brazil.

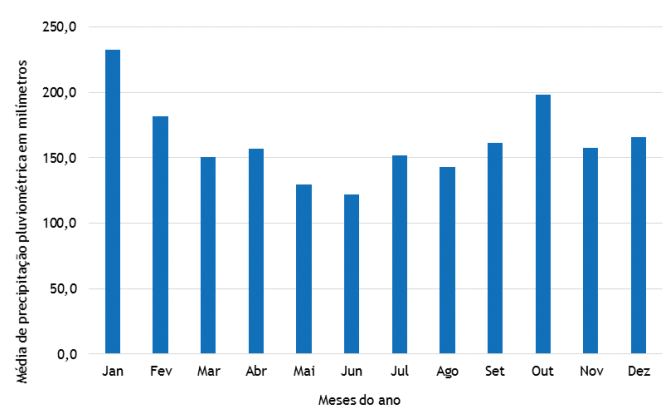

Fig. 2 - Médias mensais das precipitações pluviométricas no período de 2008 até 2012. Estado de Santa Catarina - Sul do Brasil.

Fig. 2 - Monthly averages of rainfall from 2008 to 2012. State of Santa Catarina - South of Brazil.
As taxas de incidências apresentadas na fig. 3 e na fig. 4, referem-se às doenças de síndrome diarreica aguda (SDA) e leptospirose por ano e mês. No caso das incidências anuais para a SDA, observa-se que estas apresentam as maiores taxas no ano de 2009, seguido do ano de 2008. Para as ocorrências de leptospirose foram identificados os anos de 2008 e 2011 (fig. 3).

No que se refere aos meses dos anos, as incidências da SDA, apresentam-se de forma mais expressiva nos meses de janeiro, outubro, dezembro, fevereiro, julho e abril. Para a incidência de leptospirose por mês destacam-se os meses de janeiro, outubro, dezembro, fevereiro, julho, abril e setembro (fig.4).

Com base na fig. 1 , fig. 2 , fig. 3 e na fig. 4 fica claro que nos meses onde a média de precipitação pluviométrica foi mais elevada, as incidências também foram relevantes, ou seja, é possível mencionar uma relação existente entre as ocorrências de precipitações pluviométricas elevadas com os casos de SDA e leptospirose, segundo os dados apresentados.

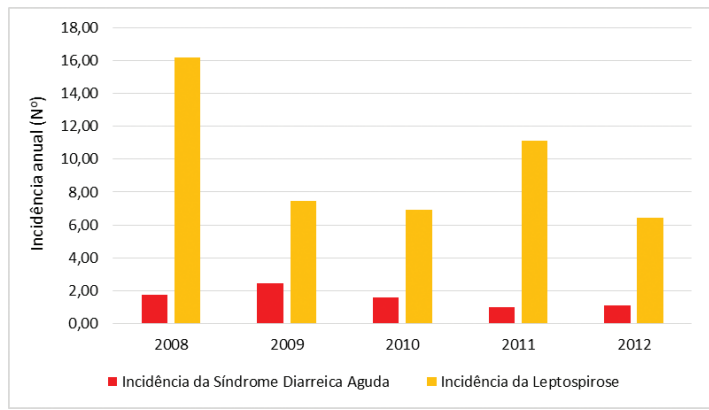

Fig. 3 - Incidências anuais da Síndrome Diarreica Aguda e da Leptospirose no período de 2008 até 2012, por 100.000 habitantes. Estado de Santa Catarina - Sul do Brasil.

Fig. 3 - Annual incidences of acute diarrheal syndrome and leptospirosis from 2008 until 2012, by 100,000 population. State of Santa Catarina - South of Brazil.

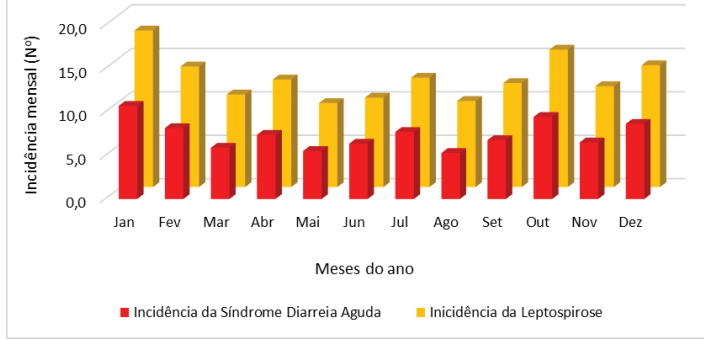

Fig. 4 - Incidências mensais da Síndrome Diarreica Aguda e da Leptospirose no período de 2008 até 2012, por 100.000 habitantes. Estado de Santa Catarina - Sul do Brasil.

Fig. 4 - Monthly incidences of acute diarrheal syndrome and leptospirosis from 2008 until 2012, by 100,000 population. State of Santa Catarina - South of Brazil. 
A análise do risco relativo foi estimada mês a mês para a ocorrência da SDA, da leptospirose e da precipitação pluviométrica com base ao mês anterior aos casos das doenças. Portanto, identificou-se o mês de junho com a menor média mensal de precipitação pluviométrica, conforme pode ser observado na fig. 2. Os riscos relativos (RR) identificados para a SDA demonstram que sua maior significância se deu nos meses de março (RR = $2,38)$, janeiro $(R R=1,93)$ e novembro $(R R=1,79)$. Para a leptospirose os riscos relativos significativos foram encontrados nos seguintes meses: dezembro ( $R R=6,05)$, fevereiro $(R R=4,14)$, janeiro $(R R=3,38)$ e abril $(R R=$ 2,11).

Nesta pesquisa o risco relativo demonstra as chances que a SDA ou a leptospirose tem de ocorrer em períodos de precipitações pluviométricas intensas ou na ocorrência de inundações.

Optou-se em demonstrar neste artigo a distribuição espacial das doenças (municípios notificados) com as ocorrências de inundações referentes ao ano de 2008. Os dados de inundações apresentados na fig. 5 e na fig. 6 encontram-se divididos em 4 classes que demonstram os totais de inundações ocorridos no ano de 2008, variando do tom mais claro de azul (classe 1 = uma ocorrência de inundação) ao mais escuro (classe 4 = quatro ocorrências de inundações). Em relação as ocorrências da Síndrome Diarreica Aguda e Leptospirose estas encontram-se divididas em 5 classes, por tamanhos de círculos, que demonstram a quantidade de ocorrência das doenças analisadas para o ano de 2008. Na fig. 5 encontram-se a distribuição dos casos de SDA com as inundações, observando os locais de notificação da SDA, com destaque para as áreas de inundações.

$\mathrm{Na}$ fig.6 é possível observar que os municípios com notificações por leptospirose concentram-se nas áreas onde houveram ocorrências de inundações.

\section{Conclusões}

A presente pesquisa foi motivada pelo elevado número de casos de doenças que são registrados a cada ocorrência de inundações. Os riscos à saúde pública em ocorrências de desastres, se não geridos com eficiência, podem representar elevados prejuízos sociais e econômicos.

Esta pesquisa vislumbrou a identificação dos municípios onde foram registrados os maiores números de casos da síndrome diarreica aguda e da leptospirose em ocorrências de precipitações pluviométricas, bem como, dos meses do ano em que houveram mais notificações destas doenças.

As informações geradas servem de ferramentas para o planejamento de decisões a serem tomadas no processo da gestão de risco prospectiva, esta que objetiva a minimização dos erros passados.

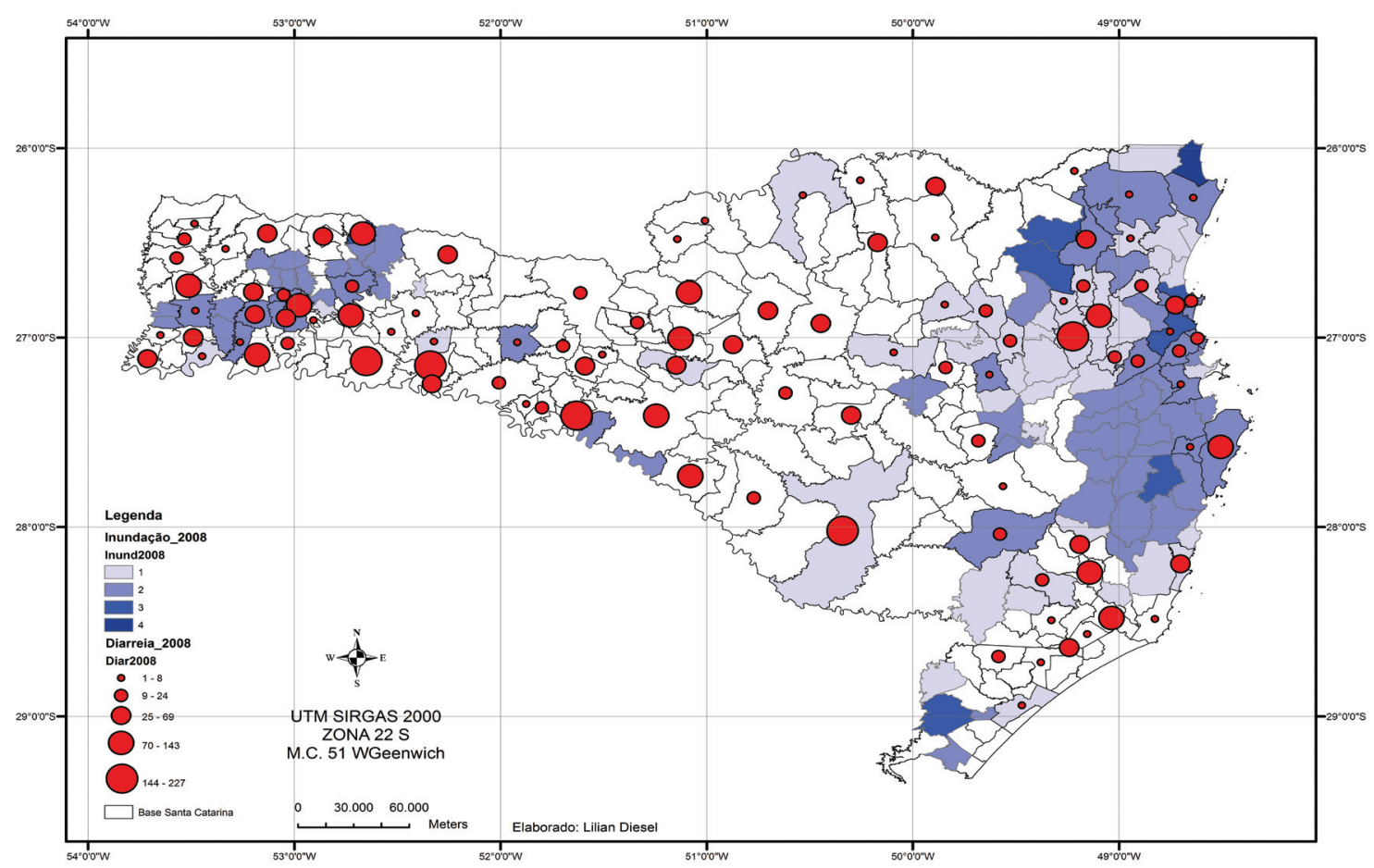

Fig. 5 - Distribuição espacial de inundações e casos de síndrome diarreica aguda no estado de Santa Catarina no ano de 2008.

Fig. 5 - Spatial distribution of floods and cases of acute diarrheal syndrome in the state of Santa Catarina in 2008. 


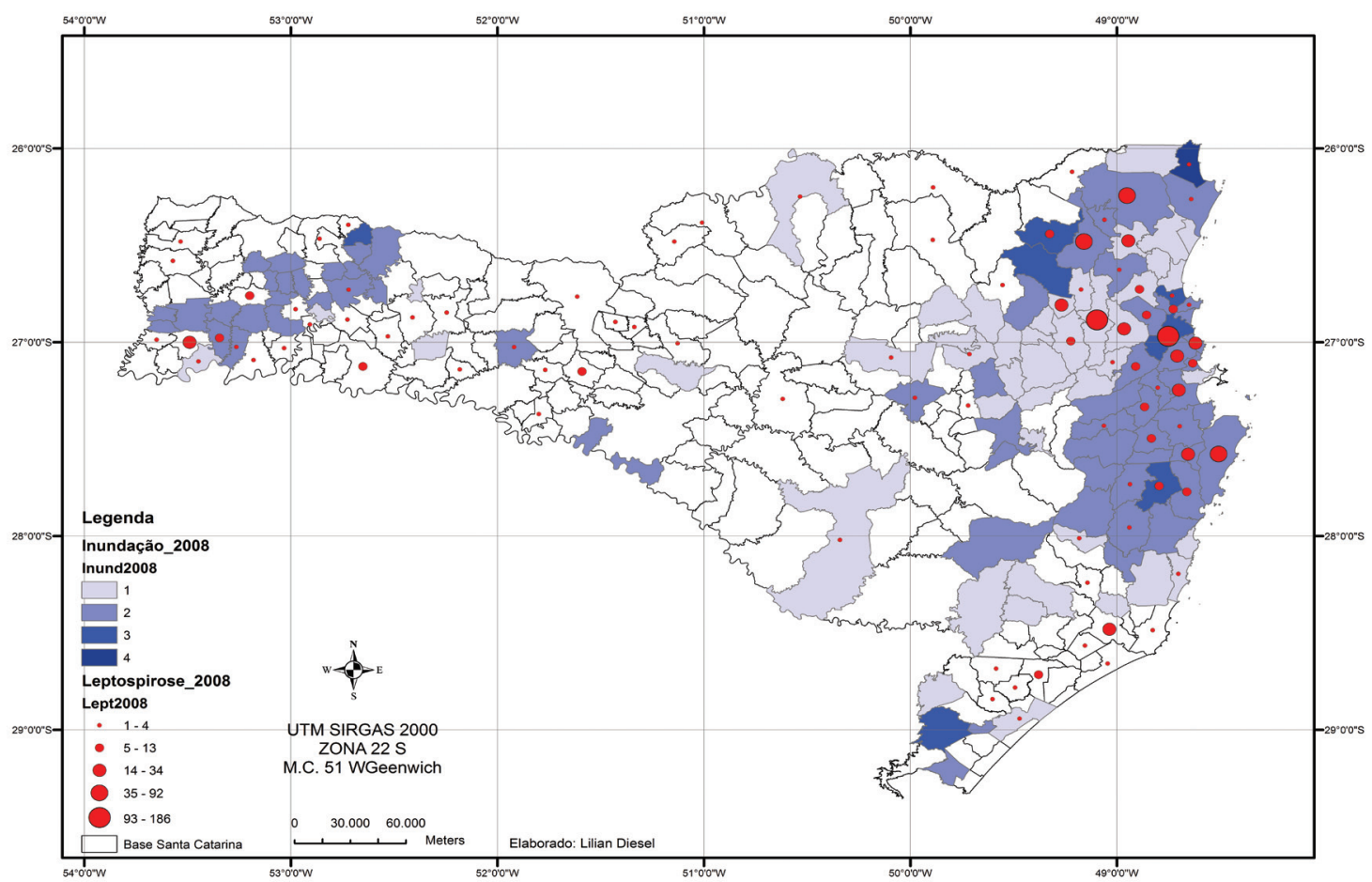

Fig. 6 - Distribuição espacial de inundações e casos de leptospirose no estado de Santa Catarina no ano de 2008.

Fig. 6 - Spatial distribution of leptospirosis cases and floods in the state of Santa Catarina in 2008.

\section{Referências bibliográficas}

Agredo, Liliana Vargas (2011). Control físico urbano para la gestión del riesgo. Bitacora, $n .^{\circ} 19$, (2) 111-122.

Castro, A. L. C. (2003). Manual de Desastres: desastres naturais. Brasília: Ministério da Integração Nacional. 174 p.

Cerutti, Dulce Fátima, Oliveira, Mara Lúcia Carneiro (2011). Aplicação da gestão de risco de desastres no Sistema Único de Saúde (SUS). Cadernos de Saúde Coletiva. Rio de Janeiro, 19 (4): 417-24.

Diesel, Lilian Elizabeth (2013). Sistema de gestão de riscos viários com o uso da geointeligência: os deslizamentos e as inundaçôes em rodovias do estado de Santa Catarina. Revista Territorium $n^{\circ}$. 20, 77-83. Disponível em: http://www. uc.pt/fluc/nicif/riscos/Documentacao/ Territorium/T20_artigos/T20_Artigo07.pdf.

Fagundes, Rodrigo da Silva (2015). Inundação e casos de síndrome diarreica aguda no estado de Santa Catarina entre 2008 - 2012 (Trabalho de conclusão do curso de medicina). Univerdade Federal de Santa Catarina.

Freitas, Maria Isabel Castreghini \& Cunha, Lúcio. (2013). Cartografia da vulnerabilidade socio-ambiental: convergências e divergências a partir de algumas experiências em Portugal e no Brasil. urbe. Revista Brasileira de Gestão Urbana, 5(1), 1531. Recuperado em 15 de setembro de 2015, de http://www.scielo.br/scielo.php?script=sci arttext\&pid=S2175-33692013000100003\&lng=pt \&tlng=pt. 10.7213/urbe.7783.

Fuentes-Vallejo, Mauricio, Higuera-Mendieta, Diana Rocío, García-Betancourt, Tatiana, Alcalá-Espinosa, Lucas Andrés, García-Sánchez, Diana, Munévar-Cagigas, David Alejandro, Brochero, Helena Luisa, González-Uribe, Catalina, \& Quintero, Juliana. (2015). Territorial analysis of Aedes aegypti distribution in two Colombian cities: a chorematic and ecosystem approach. Cadernos de Saúde Pública, 31(3), 517-530. Retrieved September 30, 2015, from http://www.scielo.br/ scielo. php? script=sci arttext\&pid=S0102$311 \times 2015000300517 \& \operatorname{lng}=$ en \& $\operatorname{tlng}=$. 10.1590/0102-311X00057214.

Guimarães, Raphael Mendonça, Cruz, Oswaldo Gonçalves, Parreira, Viviane Gomes, Mazoto, Maíra Lopes, Vieira, Juliana Dias, \& Asmus, Carmen Ildes Rodrigues Fróes. (2014). Análise temporal da relação entre leptospirose e ocorrencia de inundações por chuvas no município do Rio de Janeiro, Brasil, 2007-2012. Ciência \& Saúde Coletiva, 19(9), 3683-3692. Retrieved September 30, 2015, from http:// /www.scielo. $\mathrm{br} /$ scielo.php?script=sci_arttext \&pid=S1413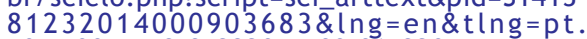 $10.1590 / 1413-81232014199.06432014$.

Lakatos, E. M., Marcon, M. de A. (2007). Metodologia cientifica: ciência e conhecimento cientifico, métodos científicos, teoria, hipóteses e variáveis. 5. ed. rev. e ampl. São Paulo: Atlas, 312p.

Souza, Elizabeth Maria Feitosa da Rocha de, Cruz, Carla Bernadete Madureira, \& Richter, Monika. (2014). O uso de geotecnologias em sistemas de transportes e organização urbana no Brasil. Mercator (Fortaleza), 13(1), 143-152. Recuperado em 15 de setembro de 2015, de http:// www. scielo.br/scielo.php?script=sci arttext\&pid=S1984-22012014000100143\&lng $=\bar{p}$ t\&tlng=pt. 10.4215/RM2014.1301.0011.

Vergara, S. C. (2007). Projetos e relatórios de pesquisa em administração. 9. ed São Paulo: Atlas, 92p.

Veyret, Y. (2007). Os Riscos - o Homem como Agressor e Vítima do Meio-ambiente. São Paulo, ed. Contexto.

Winckler, Danilo (2015). Inundação e casos de leptospirose em Santa Catarina entre 2009 - 2012 (Trabalho de conclusão do curso de medicina). Univerdade Federal de Santa Catarina. 

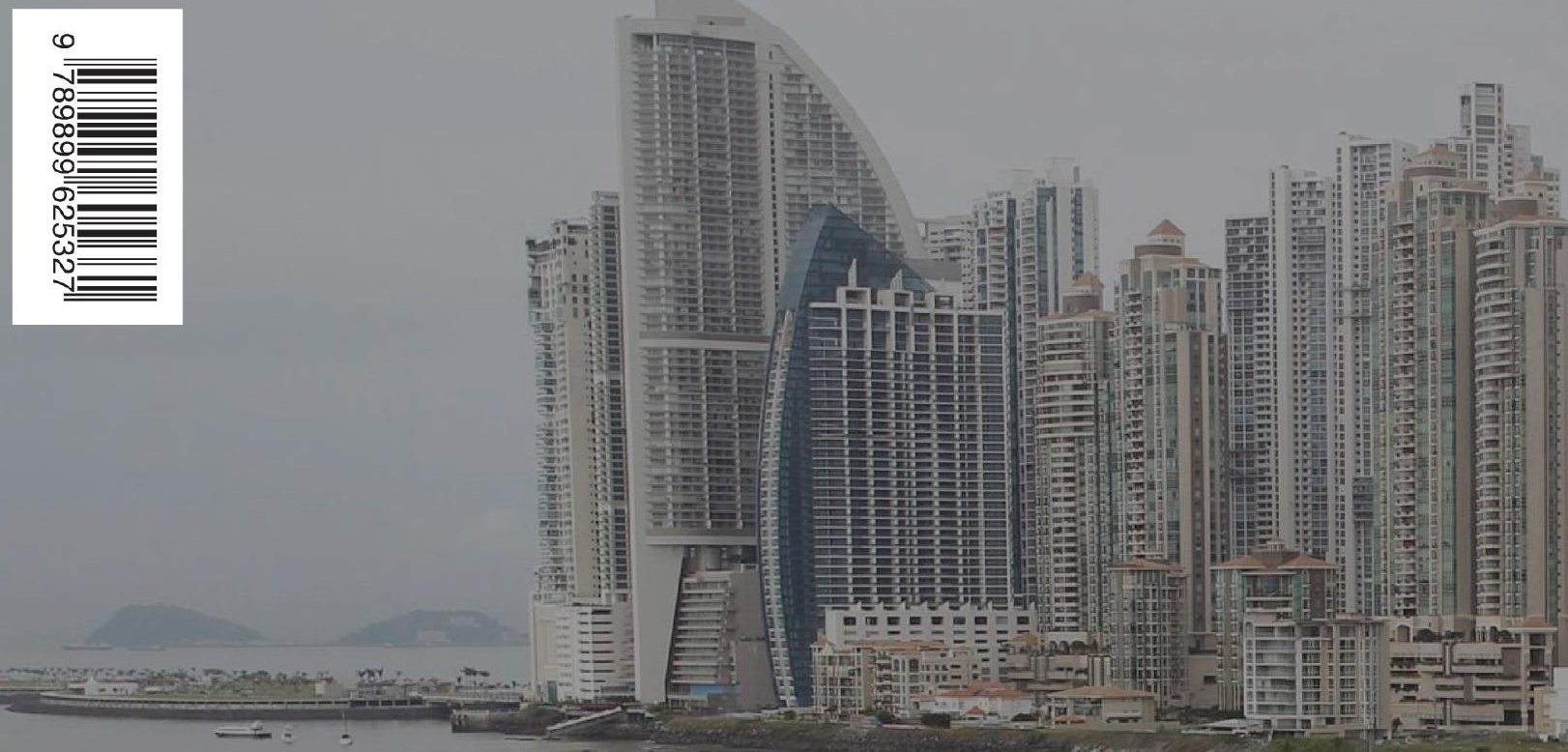

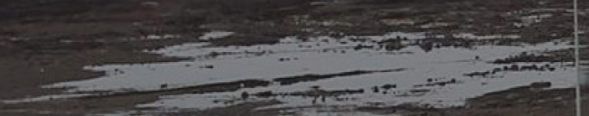

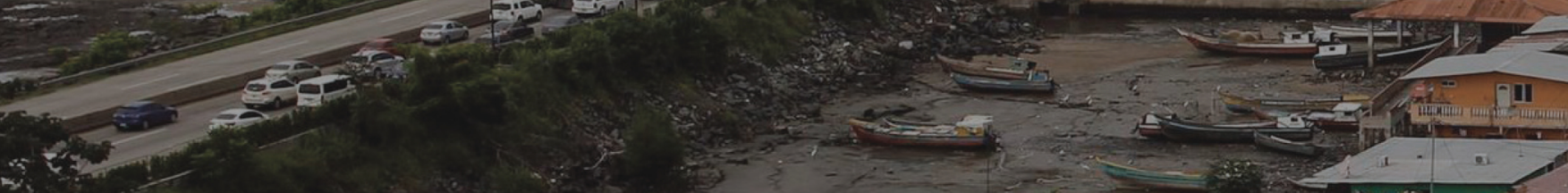

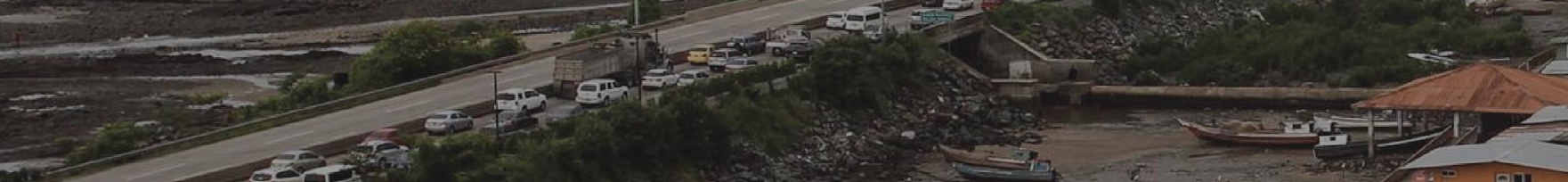
inse- $n=$ Riscos I I I

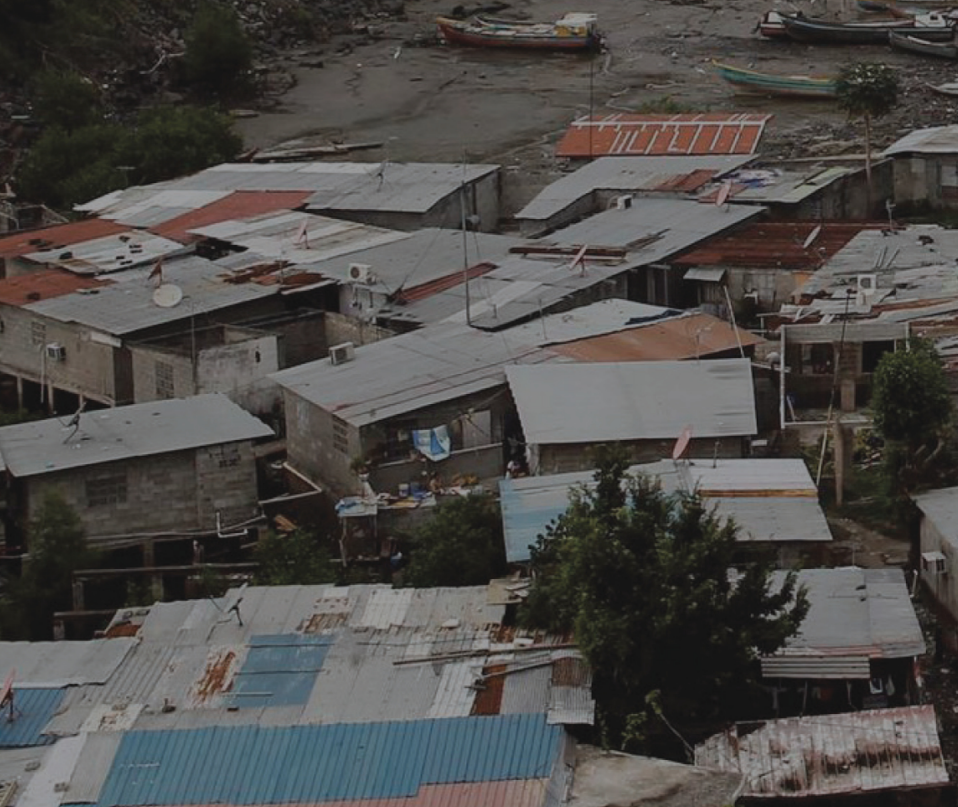

